\section{Hemolytic transfusion reactions in sickle cell disease: underappreciated and potentially fatal}

\author{
Swee Lay Thein, ${ }^{1}$ France Pirenne, ${ }^{2,3}$ Ross M. Fasano, ${ }^{4,5}$ Anoosha Habibi, ${ }^{3,6}$ \\ Pablo Bartolucci, ${ }^{6}$ Satheesh Chonat, ${ }^{5}$ Jeanne E. Hendrickson ${ }^{7}$ \\ and Sean R. Stowell ${ }^{4}$
}

${ }^{1}$ National Heart, Lung and Blood Institute, National Institutes of Health, Bethesda, MA, USA; '2Etablissement Français du Sang, INSERM U955, Université Paris Est Créteil, Créteil, France; ${ }^{3}$ Laboratoire d'Excellence GR-Ex, Paris, France; ${ }^{4}$ Center for Transfusion Medicine and Cellular Therapies, Department of Laboratory Medicine and Pathology, Emory University School of Medicine, Atlanta, GA, USA; ${ }^{5}$ Aflac Cancer and Blood Disorders Center, Department of Pediatrics, Emory University School of Medicine, Atlanta, GA, USA; 'Sickle Cell Referral Center, Department of Internal Medicine, Henri-Mondor University HospitalUPEC, AP-HP, Créteil, France and ${ }^{7}$ Departments of Laboratory Medicine and Pediatrics, Yale University School of Medicine, New Haven, CT, USA

\section{Introduction}

Patients with sickle cell disease (SCD) often receive red blood cell (RBC) transfusion support for the prevention and management of many acute and chronic disease complications..$^{1-3}$ The beneficial effects of transfusion therapy observed in recent clinical studies, and the lack of effective treatments for this population of patients, have led to an increased use of blood. ${ }^{4}$ While RBC transfusions may be life-saving, we are concerned about their expanding use and would like to raise awareness of RBC alloimmunization, a major complication of transfusion, particularly in patients with SCD in whom the incidence is much higher than in other groups of patients. ${ }^{5}$ Hemolytic transfusion reactions, which primarily occur in RBC alloimmunized patients, are often under-recognized in patients with SCD, in particular because the symptoms mimic those of acute vaso-occlusive crises, and serological markers of new alloantibodies may be equivocal., ${ }^{6,7}$ In addition to increasing the risk of potentially fatal acute or delayed-type hemolytic transfusion reactions (DHTR), ${ }^{8-10}$ the development of $\mathrm{RBC}$ alloantibodies can also significantly delay the procurement of compatible RBC for future transfusions. ${ }^{11}$

Currently, there is a lack of evidence in this area to inform best practice, and management is often based on anecdotal case reports. While there have been reports of a variety of cases illustrating the challenges associated with recognizing and treating hemolytic transfusion reactions in patients with SCD, ${ }^{12,13}$ the potential reasons for the higher incidence of RBC alloantibodies in SCD patients merit discussion. Here, we share our experience in managing alloimmunized patients and hemolytic transfusion reactions, and challenge the medical community to consider lessons learned from diagnostic criteria and mitigation policies for transfusion-related acute lung injury (TRALI) in order to minimize the morbidity and mortality associated with transfusion in patients with SCD.

\section{Why are patients with sickle cell disease at high risk of red blood cell} alloimmunization?

One possible reason for the relatively high incidence of alloimmunization observed in patients with SCD is the mismatch in RBC antigens expressed in the donor pool (primarily Northern European descent) and patients with SCD (mainly of African descent). ${ }^{8}$ Mismatch of $\mathrm{RBC}$ antigens is not the only reason, however, as a significant proportion of patients with SCD who receive phenotypically matched blood from exclusively ethnically matched donors still become alloimmunized. ${ }^{9}$ Molecular analyses of the $R H$ genes in patients with SCD and African-American donors reveal remarkable $R H$ allelic diversity in this population, with mismatch between serological Rh phenotype and RHD or RHCE genotype due to variant $R H$ alleles in a large proportion of the individuals. ${ }^{10}$ Thus, $R H$ genotyping in addition to serological typing may be required to identify the most compatible RBC, though it is not yet known if such an approach completed prospectively instead of reactively (after antibodies against alloantigens in the $R H$ family form) will decrease RBC alloimmunization, and whether it will be possible to source rarer $R H$ genotypes on a regular basis for patients on a transfusion program. The clinical context of RBC transfusion in SCD may also contribute to the higher rate of alloimmunization; the
Ferrata Storti Foundation

\section{Correspondence:}

SEAN R. STOWELL

srstowe@emory.edu

Received: August 26, 2019.

Accepted: December 18, 2019.

Pre-published: February 6, 2020.

doi:10.3324/haematol.2019.224709

Check the online version for the most updated information on this article, online supplements, and information on authorship \& disclosures: www.haematologica.org/content/105/3/539

(C)2020 Ferrata Storti Foundation

Material published in Haematologica is covered by copyright. All rights are reserved to the Ferrata Storti Foundation. Use of published material is allowed under the following terms and conditions:

https://creativecommons.org/licenses/by-nc/4.0/legalcode. Copies of published material are allowed for personal or internal use. Sharing published material for non-commercial purposes is subject to the following conditions:

https://creativecommons.org/licenses/by-nc/4.0/legalcode, sect. 3. Reproducing and sharing published material for commercial purposes is not allowed without permission in writing from the publisher. 
risk of alloimmunization in SCD is increased when patients are transfused for acute complications, such as acute chest syndrome, acute pain and acute multi-system organ failure, which are clinical complications marked by significant inflammation. Thus, unique aspects of transfusion therapy in patients with SCD, in conjunction with other possible immune perturbations, appear to place such patients at a particular risk of RBC alloimmunization. ${ }^{5,14-17}$

\section{Definitions of acute and delayed hemolytic transfusion} reactions and hyperhemolysis

While acute hemolytic transfusion reactions can largely be avoided by stringent alloantibody investigations prior to transfusion, DHTR, which typically occur days or weeks following the implicated transfusion episode of seemingly compatible $\mathrm{RBC}^{7}$ are more difficult to avoid. The delayed nature of DHTR is thought to reflect the recrudescence of an alloantibody not detected at the time of the RBC compatibility testing just prior to transfusion. ${ }^{6,18,19}$ The inability to detect RBC alloantibodies at the time of transfusion presumably reflects evanescence of a prior alloantibody response to a level below the detection threshold in routine clinical assays. Following re-exposure to the implicated alloantigen, immunological memory generated during the primary encounter facilitates an amnestic immune response that results in the rapid production of alloantibodies against the transfused unit (Figure 1). This in turn causes destruction of the transfused RBC, which is often accompanied by clinical symptoms associated with accelerated hemolysis. ${ }^{18,19}$ DHTR

A

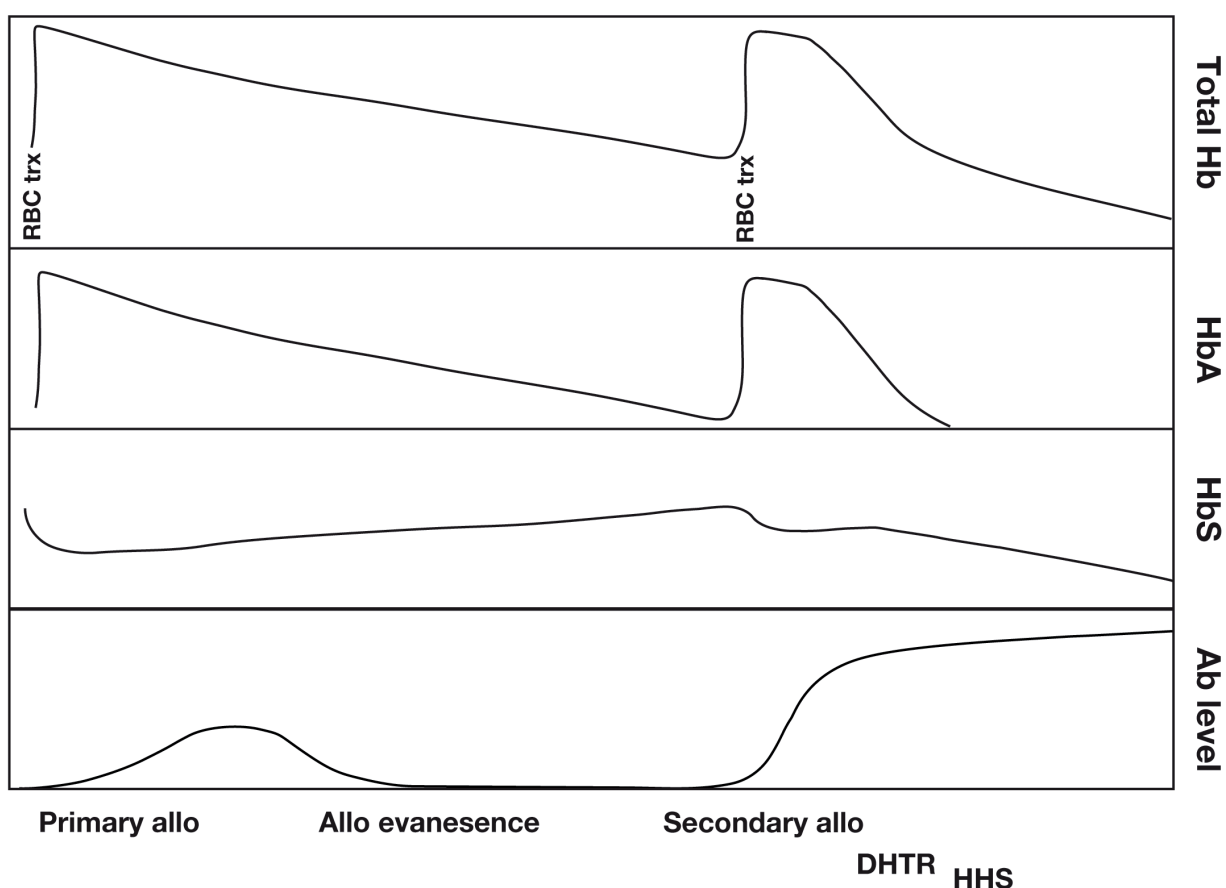

B

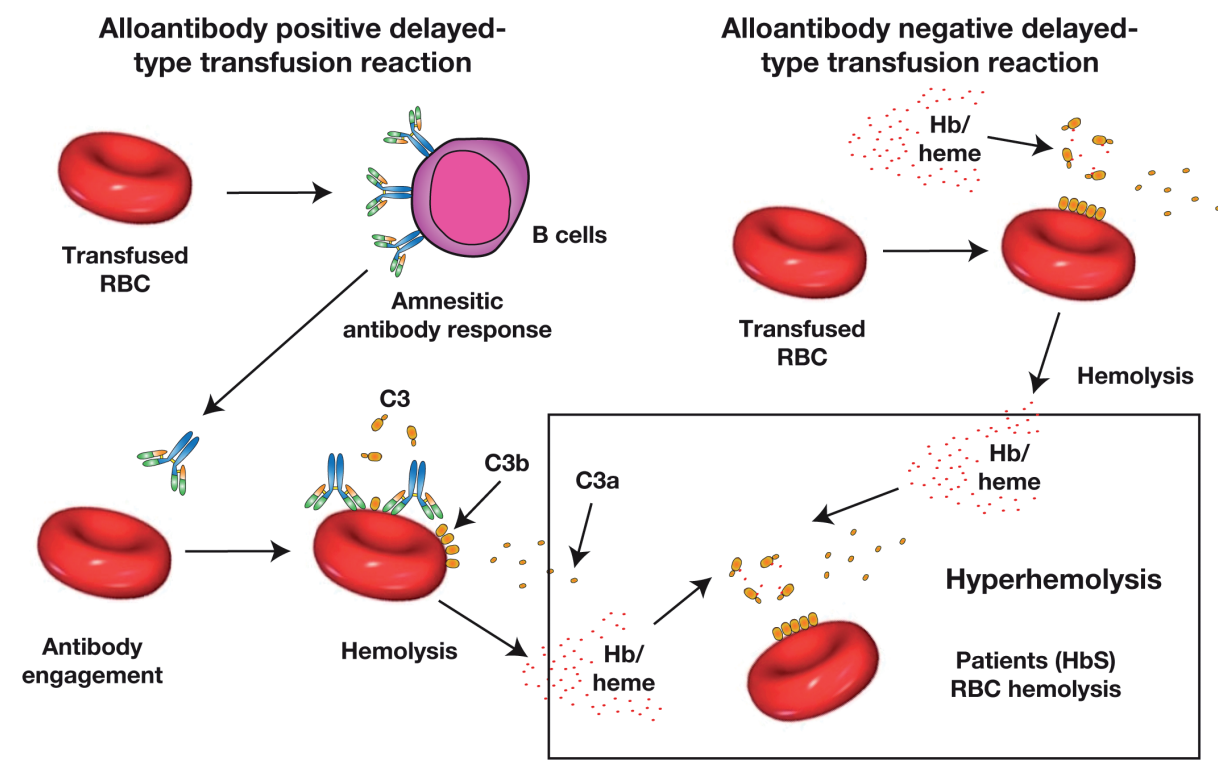

Figure 1. Delayed-type hemolytic transfusion reactions. (A) Exposure to a red blood cell (RBC) alloantigen through transfusion or pregnancy can result in the development of alloantibodies (allo) that quickly evanescence over time, possibly preventing their detection prior to a subsequent transfusion. Re-exposure to RBC expressing the same alloantigen can induce an amnestic alloantibody response, which can cause accelerated clearance of the transfused RBC resulting in hemolysis and a delayed-type transfusion reaction (DHTR). Alloantibody induced clearance of transfused RBC can occasionally result in hyperhemolysis, otherwise known as hyperhemolytic syndrome (HHS), which is signified by the accelerated clearance of the patient's own RBC and which can be particularly fatal. (B). Alloantibodies that develop in response to exposure to alloantigens can lead to direct clearance of RBC through a variety of antibody effector mechanisms, including complement activation. Sometimes patients will experience a DHTR in the absence of a detectable alloantibody; an alloantibody may be present and simply be below the detection threshold of clinical assays or an alloantibody may be absent entirely, with the DHTR possibly reflecting heme-mediated complement activation and RBC hemolysis. Regardless of the mode of hemolysis experienced by transfused RBC in the setting of a DHTR, heme released may activate complement, thereby potentially contributing to the development of hyperhemolysis. Trx: transfusion; Hb: hemoglobin. 
can be further complicated by bystander hemolysis or hyperhemolysis, a process that results in the accelerated clearance of both the transfused RBC and the patient's own $\mathrm{RBC}^{20,21}$ (Figure 1). Such hyperhemolysis can be particularly fatal in patients with SCD for reasons that remain incompletely understood.

\section{Attention to a high incidence of delayed hemolytic transfusion reactions}

While DHTR can be life-threatening, unawareness of their frequency and lack of severity of these transfusion reactions have likely resulted in too little attention regarding their potential impact on overall SCD morbidity and mortality. For years, the overall incidence of DHTR per transfusion in SCD was estimated to be around 1:1000.22-24 making it a relatively uncommon transfusion reaction in this population of patients. However, newer reports suggest that these data may be misleading. As mentioned previously, given the similarities between the clinical presentation of DHTR and the more common complications of vaso-occlusive crises, DHTR can be easily missed. ${ }^{6,7,25}$ Unless an alloantibody screen is performed, an amnestic alloantibody response will not be detected and a diagnosis of DHTR may not be entertained. Furthermore, as some reports suggest that as many as $30 \%$ of DHTR can be alloantibody-negative, ${ }^{14,26}$ clinicians must rely on $\mathrm{HbA}$ measurements obtained within $48 \mathrm{~h}$ of the implicated transfusion and at the time of a presumptive DHTR in order to make a reliable diagnosis of $\mathrm{DHTR}^{12,27}$ (Figure 1). Unfortunately, alloantibody screens and $\mathrm{HbA}$ values are not routinely ordered following transfusion or in recently transfused patients admitted for acute pain, raising the possibility that the incidence of DHTR in patients with SCD may be much higher than previously appreciated. ${ }^{27}$

In an effort to assess the incidence of DHTR more accurately, a recent prospective study evaluated adult patients after transfusion using total $\mathrm{Hb}, \mathrm{HbA}$ and $\mathrm{HbS}$ quantification within $48 \mathrm{~h}$ after all transfusions and defined DHTR as a significant decrease in $\mathrm{HbA}(>50 \%)$ and/or in total $\mathrm{Hb}$ levels $(>30 \%)$ within 25 days of a trigger transfusion along with hemoglobinuria, symptoms of a vaso-occlusive crisis, and/or worsening symptoms of anemia. ${ }^{27}$ Using this approach, a DHTR was found to occur following $4.2 \%$ of episodic transfusions, ${ }^{27}$ over ten times more frequently than previously speculated, making DHTR the single most common adverse event following episodic transfusion in patients with SCD. Nearly $11 \%$ of all patients with DHTR died, ${ }^{27}$ suggesting that these reactions are not only more common than previously suggested, but also likely to affect SCD mortality significantly. These results provide one possible explanation for the recent observation that alloimmunized patients with SCD have a much higher mortality rate than non-alloimmunized individuals with SCD. ${ }^{28}$

\section{Comparison of delayed hemolytic transfusion reactions with transfusion-related acute lung injury}

There are resemblances between the underappreciated incidence and impact of DHTR in patients with SCD and the history of other transfusion reactions with fatal outcomes. ${ }^{29}$ Until relatively recently, TRALI was a rarely recognized complication of primarily platelet and plasma transfusion defined by acute lung injury within $6 \mathrm{~h}$ of transfusion in the presence of hypoxia, with radiographic evidence of bilateral infiltrates in the absence of circulato- ry overload. ${ }^{30}$ Because patients who are susceptible to TRALI often have significant comorbidities, changes in pulmonary function that accompanied transfusion were historically attributed to other etiologies. ${ }^{31}$ However, once the impact of TRALI was recognized and regulatory agencies heightened hemovigilance efforts, TRALI quickly became identified as the most common cause of transfusion-related mortality in the USA and Europe. ${ }^{32}$ Epidemiological studies found associations between blood products donated from multiparous women and other donor factors that increased the likelihood that a recipient would develop TRALI. ${ }^{32}$ In particular, anti-HLA and other anti-leukocyte alloantibodies that may bind and activate leukocytes intravascularly became implicated in the pathogenesis of TRALI. ${ }^{33-35}$ Implementation of manufacturing practices that excluded multiparous females and other donors who appeared to increase the risk of TRALI in transfusion recipients has resulted in a significant reduction in TRALI cases. ${ }^{36,37}$ Thus, TRALI provides a key example of an underappreciated transfusion complication that can result in significant morbidity and mortality and that, upon additional study, improved diagnostic criteria and changes in clinical practice, has dramatically reduced in incidence over time. ${ }^{31}$

\section{Strategies to prevent delayed hemolytic transfusion reactions}

The most effective way to prevent a DTHR is to avoid unnecessary RBC transfusion. When transfusion avoidance is not feasible, provision of the most compatible $\mathrm{RBC}$ units is recommended. In an effort to reduce the risk of DHTR, transfusion services keep records of previously identified alloantibodies in order to reduce the risk of reexposure to a particular alloantigen once an alloantibody has been detected. Using this approach, hospital transfusion services provide RBC that are negative for the particular alloantigens against which a patient has previously made alloantibodies. However, when patients seek care in multiple healthcare systems this information is often not available. ${ }^{38,39}$ In this setting, transfusion services can only rely on transfusion histories at the presenting facility or other facilities if obtainable, which are often either not obtained or incomplete. ${ }^{38,39}$ As this approach often results in inadequate alloimmunization histories, ${ }^{40}$ multiple encounters in different healthcare systems place patients with SCD at a significantly high risk of DHTR. ${ }^{38}$ Furthermore, transfusions are often initiated when patients present with acute complications at centers where providers may not be familiar with SCD management, including transfusion complications. Implementation of healthcare-wide acute care plans for patients with SCD that include consideration of possible transfusion complications may provide the type of guidance needed to increase awareness among all providers within a healthcare system.

Nonetheless, despite effective communication between healthcare systems, alloantibodies may not be detected if antibody evaluations are not routinely completed following transfusion episodes that are at a higher risk of inducing alloantibodies. This reflects the ability of newly formed alloantibodies to fall below the level of detection prior to a subsequent transfusion evaluation, which may occur months to years later. ${ }^{41}$ Thus, RBC alloantibody evanescence can contribute significantly to the risk of DHTR in the absence of systematic post-transfusion sero- 
logical evaluation. To avoid these challenges, routine serological testing should be considered for all SCD patients 1 to 3 months after each transfusion episode..$^{42}$ Even with such a policy in place, up to $30 \%$ of DHTR with bystander hemolysis occur in the absence of any detectable alloantibody, ${ }^{14,26}$ making it particularly difficult to predict and prevent these transfusion reactions fully.

\section{Shared laboratory data between hospital systems is critical}

Significant barriers to laboratory information exchange exist in many healthcare systems in many countries, and efforts that allow alloantibody identification histories of all patients (including those with SCD) to be shared between such systems must become a priority. This critical patient safety initiative would facilitate antibody identification in a patient's sample, would decrease the burden of identifying optimal RBC units when working with incomplete transfusion histories, would increase the timely provision of fully compatible RBC units, and would decrease the incidence of DHTR. ${ }^{38,39}$ Currently, the lack of such data sharing prevents many transfusion services from knowing the transfusion requirements of newly encountered patients with SCD at the time of a transfusion request ${ }^{40}$ or from knowing the availability of compatible RBC once alloantibodies have been characterized. ${ }^{11,43}$ If complex alloantibody profiles were known a priori and corresponding donor databases were available, compatible donor units could be readily identified, allowing transfusion requirements to be addressed in a more timely and safe manner. ${ }^{38,39,44}$

\section{Routine post-transfusion antibody screening and $\mathrm{HbA}$ quantification should be considered to improve identification of red blood cell alloantibodies and delayed hemolytic transfusion reactions}

Although additional studies are certainly needed to establish the incidence of DHTR in various hospital settings, more uniform detection of these reactions is important if future DHTR are to be avoided and if effective treatment strategies are to be implemented ${ }^{45}$ While patients may experience accelerated clearance of transfused RBC in the absence of clinical systems, to facilitate detection of clinically meaningful DHTR, we recommend that patients with SCD with a history of transfusion in the preceding 21 days who present with any complication requiring medical attention should be evaluated with an antibody screen regardless of whether or not another RBC transfusion is warranted. Using this approach, a higher percentage of alloantibodies that form as a result of a recent transfusion should be identified. However, as nearly $30 \%$ of DHTR have been reported to occur in the absence of detectable alloantibodies, ${ }^{14,26}$ evaluation for DHTR will also require acquisition of $\mathrm{HbA}$ values following transfusion and at the time of clinical presentation of any complication requiring medical attention. ${ }^{27}$ As episodic transfusions are much more likely to be initiated during acute complications and result in DHTR, ${ }^{6,7,27}$ polices that include obtaining $\mathrm{HbA}$ values within $48 \mathrm{~h}$ following an episodic transfusion may be particularly helpful when interpreting a $\mathrm{HbA}$ value at the time of suspected DHTR. Thus, we recommend considering routine $\mathrm{HbA}$ measurements following any episodic RBC transfusion and an antibody screen and $\mathrm{HbA}$ measurement at the time of any hospital presentation within 21 days of the most recent episodic transfusion (Table 1).

\section{Therapeutic options for ongoing delayed hemolytic transfusion reactions}

In addition to facilitating more accurate diagnoses of DHTR, routine approaches aimed at identifying DHTR will allow consideration of more effective therapeutic options for ongoing DTHR; these therapies are particularly important to consider for DHTR involving hyperhemolysis. Erythropoietin and intravenous iron are often given to boost endogenous $\mathrm{RBC}$ production in a setting of severe anemia. ${ }^{12}$ Plasmapheresis has also been attempted

Table 1. Recommendations for better prevention, more accurate diagnosis and improved treatment of delayed-type hemolytic transfusion reactions in sickle cell disease.

\section{Prevention:}

National and International

1) RBC alloimmunization databases

2) RBC donor databases

Institutional

1) Reduce RBC alloimmunization through prophylactic matching for Rh (C/c, E/e) and $K$ antigens.

2) Judicious use of RBC transfusions

3) Routine alloantibody evaluation for all patients with SCD within 1 to 3 months after each episodic transfusion (while rare, some alloantibodies may not be detectable within 3 months after transfusion)

\section{Diagnosis:}

Institutional

1) Alloantibody screen for any SCD exacerbation within 21 days of transfusion (regardless of whether another transfusion is being considered)

2) $\mathrm{HbA}$ quantification within $48 \mathrm{~h}$ following episodic transfusion and at the time of any SCD exacerbation occurring within 21 days of a transfusion

\section{Treatment:}

Institutional

1) Supportive care (including erythropoietin and iron)

2) Intravenous immunoglobin and corticosteroids

3) Consideration of treatment with complement inhibitors in cases of severe DHTR (including those with hyperhemolysis)

4) Consider rituximab prophylaxis in cases with a history of severe DHTR and only "least incompatible" blood can be sourced for transfusion.

RBC: red blood cell; SCD: sickle cell disease; HbA: hemoglobin A. 
to reduce heme levels ${ }^{46}$ although the level of anemia and inability to transfuse may prevent this from becoming a realistic option in many patients. Intravenous immunoglobulin and corticosteroids may further reduce hemolysis in the setting of DHTR ${ }^{47}$ While characteristics of the transfused unit, such as RBC storage, may have an impact on transfusion outcomes in SCD, ${ }^{48}$ recent studies suggest that exuberant complement activation may account for the most severe DHTR with accompanying hyperhemolysis. ${ }^{49}$ Consistent with this, treatment of patients experiencing DHTR-associated hyperhemolysis with eculizumab, an anti-C5 complement-blocking antibody, has been shown to reverse complement activation, reduce hemolysis, and result in rapid clinical improvement. 49,50 While additional studies are needed, these reports hold promise and suggest that more effective treatment options that could significantly improve patients' care may be on the horizon (Table 1).

Avoiding additional RBC transfusion at the time of an ongoing DHTR with bystander hemolysis is recommended, as transfusion of even seemingly compatible RBC that are negative for all alloantigens that the patient is known to be alloimmunized against may worsen the ongoing hemolysis. If the alloantibody in question cannot be identified or if it is identified but compatible units cannot be allocated, alloantibody function tests can be ordered to assess the clinical significance of a patient's alloantibodies. This test typically involves evaluation of monocyte engulfment of antibody-coated cells in vitro as a read out of alloantibody function. ${ }^{51}$ However, this approach is time-consuming, and may not provide timely results in an acute setting. ${ }^{51}$ Should the clinical status of the patient necessitate consideration of a "least incompatible" RBC transfusion, rituximab prophylaxis has been described to reduce DHTR in small case reports. ${ }^{52,53}$

\section{Summary}

In conclusion, RBC alloantibodies and DHTR are not uncommon in patients with SCD. They are underappreciated and, in our opinion, are the single leading cause of transfusion-associated morbidity and mortality in this vulnerable population of patients. Many of the challenges associated with preventing and treating DTHR can be addressed by developing international and national RBC alloantibody databases, limiting RBC transfusions to situations that are evidence-based, implementing more accurate diagnostic strategies (through routine use of $\mathrm{HbA}$ quantification and standard antibody screening), better understanding the pathophysiology, and formally testing additional prophylactic and treatment approaches to prevent and treat these reactions. We urge our colleagues in hematology, transfusion medicine (from donor centers to transfusion services), laboratory information technology, funding agencies, and regulatory agencies to view RBC alloimmunization and DTHR in patients with SCD with a similar urgency as TRALI was viewed in past decades. Such a heightened awareness, and subsequent industry changes, are predicted to directly reduce the significant transfusionassociated complications that contribute to the current morbidity and mortality of patients with SCD.

\section{Acknowledgments}

This perspective was initially conceived as a result of a postconference formal meeting after the first international conference on delayed type transfusion reactions in Creteil, France. We thank all those who organized, supported and attended this conference, while particularly acknowledging those who directly participated in the post meeting and subsequent discussions on this topic, including Karina Yazdanbakhsh, Armand Mekontso Dessap, Lubka Roumenina, Eldad Hod and Chris Tormey.

\section{References}

1. Adams RJ, McKie VC, Hsu L, et al. Prevention of a first stroke by transfusions in children with sickle cell anemia and abnormal results on transcranial Doppler ultrasonography. N Engl J Med. 1998;339(1):5-11.

2. Platt OS. Prevention and management of stroke in sickle cell anemia. Hematology Am Soc Hematol Educ Program. 2006:54-57.

3. Howard J. Sickle cell disease: when and how to transfuse. Hematology Am Soc Hematol Educ Program. 2016;2016(1):625-31.

4. Drasar E, Igbineweka N, Vasavda N, et al. Blood transfusion usage among adults with sickle cell disease - a single institution experience over ten years. Br J Haematol. 2011;152(6):766-770.

5. Yazdanbakhsh K, Ware RE, Noizat-Pirenne F. Red blood cell alloimmunization in sickle cell disease: pathophysiology, risk factors, and transfusion management. Blood. 2012;120(3):528-537.

6. Vidler JB, Gardner K, Amenyah K, Mijovic A, Thein SL. Delayed haemolytic transfusion reaction in adults with sickle cell disease: a 5-year experience. Br J Haematol. 2015;169(5):746-53.

7. Habibi A, Mekontso-Dessap A, Guillaud C, et al. Delayed hemolytic transfusion reaction in adult sickle-cell disease: presenta- tions, outcomes, and treatments of 99 referral center episodes. Am J Hematol. 2016;91 (10):989-994.

8. Nickel RS, Hendrickson JE, Fasano RM, et al. Impact of red blood cell alloimmunization on sickle cell disease mortality: a case series. Transfusion. 2016;56(1):107-114.

9. Pirenne F, Yazdanbakhsh K. How I safely transfuse patients with sickle-cell disease and manage delayed hemolytic transfusion reactions. Blood. 2018;131(25):2773-2781.

10. Dean CL, Maier CL, Chonat S, et al. Challenges in the treatment and prevention of delayed hemolytic transfusion reactions with hyperhemolysis in sickle cell disease patients. Transfusion. 2019;59(5):16981705.

11. Vichinsky EP, Earles A, Johnson RA, Hoag MS, Williams A, Lubin B. Alloimmunization in sickle cell anemia and transfusion of racially unmatched blood. $N$ Engl J Med. 1990;322(23):1617-1621.

12. Chou ST, Jackson T, Vege S, Smith-Whitley K, Friedman DF, Westhoff CM. High prevalence of red blood cell alloimmunization in sickle cell disease despite transfusion from Rh-matched minority donors. Blood. 2013;122(6):1062-1071.

13. Chou ST, Evans P, Vege S, et al. RH genotype matching for transfusion support in sickle cell disease. Blood. 2018;132(11):1198-1207.

14. Chou ST, Fasano RM. Management of patients with sickle cell disease using trans- fusion therapy: guidelines and complications. Hematol Oncol Clin North Am. 2016;30(3):591-608.

15. Fasano RM, Booth GS, Miles M, et al. Red blood cell alloimmunization is influenced by recipient inflammatory state at time of transfusion in patients with sickle cell disease. Br J Haematol. 2015;168(2):291-300.

16. Yazdanbakhsh K. Immunoregulatory networks in sickle cell alloimmunization. Hematology Am Soc Hematol Educ Program. 2016;2016(1):457-461.

17. Platt OS. Sickle cell anemia as an inflammatory disease. J Clin Invest. 2000;106(3):3378.

18. Williams LA, 3rd, Lorenz RG, Tahir A, Pham $\mathrm{HP}$, Marques MB. High percentage of evanescent red cell antibodies in patients with sickle cell disease highlights need for a National Antibody Database. South Med J. 2016;109(9):588-591.

19. Siddon AJ, Kenney BC, Hendrickson JE, Tormey CA. Delayed haemolytic and serologic transfusion reactions: pathophysiology, treatment and prevention. Curr Opin Hematol. 2018;25(6):459-467.

20. King KE, Shirey RS, Lankiewicz MW Young-Ramsaran J, Ness PM. Delayed hemolytic transfusion reactions in sickle cell disease: simultaneous destruction of recipients' red cells. Transfusion. 1997;37(4):376381.

21. Ibanez C, Habibi A, Mekontso-Dessap A, et 
al. Anti-HI can cause a severe delayed hemolytic transfusion reaction with hyperhemolysis in sickle cell disease patients. Transfusion. 2016;56(7):1828-1833.

22. Talano JA, Hillery CA, Gottschall JL, Baylerian DM, Scott JP. Delayed hemolytic transfusion reaction/hyperhemolysis syndrome in children with sickle cell disease. Pediatrics. 2003;111(6 Pt 1):e661-665.

23. Michot JM, Driss F, Guitton C, et al. Immunohematologic tolerance of chronic transfusion exchanges with erythrocytapheresis in sickle cell disease. Transfusion. 2015;55(2):357-363.

24. Aygun B, Padmanabhan S, Paley C, Chandrasekaran V. Clinical significance of RBC alloantibodies and autoantibodies in sickle cell patients who received transfusions. Transfusion. 2002;42(1):37-43.

25. Diamond WJ, Brown FL Jr, Bitterman P, Klein HG, Davey RJ, Winslow RM. Delayed hemolytic transfusion reaction presenting as sickle-cell crisis. Ann Intern Med. 1980;93(2): 231-234.

26. Chadebech P, Habibi A, Nzouakou R, et al. Delayed hemolytic transfusion reaction in sickle cell disease patients: evidence of an emerging syndrome with suicidal red blood cell death. Transfusion. 2009;49(9):17851792.

27. Narbey D, Habibi A, Chadebech P, et al. Incidence and predictive score for delayed hemolytic transfusion reaction in adult patients with sickle cell disease. Am J Hematol. 2017;92(12):1340-1348.

28. Telen MJ, Afenyi-Annan A, Garrett ME, Combs MR, Orringer EP, Ashley-Koch AE. Alloimmunization in sickle cell disease: changing antibody specificities and association with chronic pain and decreased survival. Transfusion. 2015;55(6 Pt 2):13781387.

29. Carson IL, Triulzi DI, Ness PM. Indications for and adverse effects of red-cell transfusion. N Engl J Med. 2017;377(13): 12611272.

30. Yomtovian R, Kline W, Press C, et al. Severe pulmonary hypersensitivity associated with passive transfusion of a neutrophil-specific antibody. Lancet. 1984;1(8371):244-246.

31. Shaz BH, Stowell SR, Hillyer CD. Transfusion-related acute lung injury: from bedside to bench and back. Blood. 2011;117(5):1463-1471.
32. Silliman CC, Boshkov LK, Mehdizadehkash $Z$, et al. Transfusion-related acute lung injury: epidemiology and a prospective analysis of etiologic factors. Blood. 2003;101(2):454-462.

33. Looney MR, Su X, Van Ziffle JA, Lowell CA, Matthay MA. Neutrophils and their Fc gamma receptors are essential in a mouse model of transfusion-related acute lung injury. J Clin Invest. 2006;116(6):16151623.

34. Kapur R, Kim M, Rebetz J, Hallstrom B, et al. Gastrointestinal microbiota contributes to the development of murine transfusionrelated acute lung injury. Blood Adv. 2018;2(13):1651-1663.

35. Semple JW, Rebetz J, Kapur R. Transfusionassociated circulatory overload and transfusion-related acute lung injury. Blood. 2019;133(17):1840-1853.

36. Chapman CE, Stainsby D, Jones H, et al. Ten years of hemovigilance reports of transfusion-related acute lung injury in the United Kingdom and the impact of preferential use of male donor plasma. Transfusion. 2009;49 (3):440-452.

37. Murphy MF, Navarrete C, Massey E. Donor screening as a TRALI risk reduction strategy. Transfusion. 2009:49(9):1779-1782.

38. Unni N, Peddinghaus M, Tormey CA, Stack G. Record fragmentation due to transfusion at multiple health care facilities: a risk factor for delayed hemolytic transfusion reactions. Transfusion. 2014;54(1):98-103

39. Harm SK, Yazer MH, Monis GF, Triulzi DJ, Aubuchon JP, Delaney M. A centralized recipient database enhances the serologic safety of RBC transfusions for patients with sickle cell disease. Am J Clin Pathol. 2014;141(2):256-261

40. Fasano RM, Branscomb J, Lane PA, Josephson CD, Snyder AB, Eckman JR. Transfusion service knowledge and immunohaematological practices related to sickle cell disease and thalassemia. Transfus Med. 2019;29(3):185-192

41. Stack G, Tormey CA. Detection rate of blood group alloimmunization based on real-world testing practices and kinetics of antibody induction and evanescence. Transfusion. 2016;56(11):2662-2667.

42. Castro O, Oneal P, Medina A, Onojobi G, Gordeuk VR. Preventing delayed hemolytic transfusion reactions in sickle cell disease. Transfusion. 2016;56(11):2899-2900.
43. Dean CL, Maier CL, Roback JD, Stowell SR Multiple hemolytic transfusion reactions misinterpreted as severe vaso-occlusive crisis in a patient with sickle cell disease. Transfusion. 2019;59(29):448-453.

44. Hauser RG, Hendrickson JE, Tormey CA TRIX with treats: the considerable safety benefits of a transfusion medicine registry. Transfusion. 2019;59(8):2489-2492.

45. Mekontso Dessap A, Pirenne F, Razazi K, et al. A diagnostic nomogram for delayed hemolytic transfusion reaction in sickle cell disease. Am J Hematol. 2016;91(12):11811184.

46. Hayes C, Shafi H, Mason H, Klapper E. Successful reduction of plasma free-hemoglobin using therapeutic plasma exchange: a case report. Transfus Apher Sci. 2016;54(2): 253-255.

47. Gardner K, Hoppe C, Mijovic A, Thein SL. How we treat delayed haemolytic transfusion reactions in patients with sickle cell disease. Br J Haematol. 2015;170(6):745756.

48. Karafin MS, Carpenter E, Pan A, Simpson P, Field JJ. Older red cell units are associated with an increased incidence of infection in chronically transfused adults with sickle cell disease. Transfus Apher Sci. 2017;56(3):345351.

49. Dumas G, Habibi A Onimus $T$, et al Eculizumab salvage therapy for delayed hemolysis transfusion reaction in sickle cell disease patients. Blood. 2016;127(8):10621064

50. Chonat S, Quarmyne MO, Bennett CM, et al. Contribution of alternative complement pathway to delayed hemolytic transfusion reaction in sickle cell disease. Haematologica. 2018;103(10):e483-e485.

51. Tong TN, Cen S, Branch DR. The monocyte monolayer assay: past, present and future. Transfus Med Rev. 2019:33(1):24-28.

52. Noizat-Pirenne F, Bachir D, Chadebech P, et al. Rituximab for prevention of delayed hemolytic transfusion reaction in sickle cell disease. Haematologica. 2007;92(12):e132135.

53. Noizat-Pirenne F, Habibi A, MekontsoDessap A, et al. The use of rituximab to prevent severe delayed haemolytic transfusion reaction in immunized patients with sickle cell disease. Vox Sang. 2015;108(3): 262-267. 